\title{
Reducing Interference with Cognitive Relays
}

\author{
Abdulla Firag ${ }^{\dagger} \quad$ Peter J. Smith ${ }^{\ddagger}$ \\ ${ }^{\dagger}$ Department of Mathematics and Statistics \\ ${ }^{\ddagger}$ Department of Electrical and Computer Engineering and Mathematics and Statistics \\ University of Canterbury, Christchurch, New Zealand
}

\begin{abstract}
In this paper, we consider the interference caused by a secondary system to the primary system in a cognitive radio network. We consider a secondary system deployed in a field of primary receivers. Primary receivers are assumed to be distributed according to a Poisson point process with a density parameter $\lambda_{p}$. We assume that the secondary system consists of source, relay, and destination terminals, and the source terminal can communicate with the destination terminal either directly (direct mode) or using the relay (relaying mode). We derive analytical expressions for the outage probability of both modes including pathloss, shadowing and multipath fading, and validate the analytical results by simulation. We also derive analytical expressions for the maximum interference caused by both modes to the primary receivers. The results show that to achieve the same median SNR level, the relaying mode may cause less interference to the primary receivers than the direct mode in high pathloss and low shadowing conditions. However, the relaying mode has much higher interference than the direct mode if both modes have the same capacity performance. The results also show that the capacity of the relaying mode has to be reduced by about one half to provide the same level of interference as the direct mode in a typical channel environment.
\end{abstract}

\section{INTRODUCTION}

Recently, cognitive radio has been proposed as a means to exploit the under-utilized licensed spectrum [1], [2], [3]. The main objective of cognitive radio networks is that the quality-of-service (QoS) of the primary network should not be degraded significantly due to the presence of the secondary network. On other hand a secondary network has to provide a reasonable capacity to justify its deployment cost [4]. Hence, the cognitive radio network has the dual aim of achieving as much capacity as possible with minimum interference to the primary network. A considerable body of research is now available on cognitive radio network interference problems [5], [6], [7]. These studies show that unlike conventional licensed networks, the capacity of a secondary network is significantly affected by the coexisting primary system due to the fact that the transmission power of the secondary system is limited by the primary network requirements for QoS.

Relays are commonly used in wireless communication to increase range or reduce transmission power at the transmitter [8], [9]. The relaying terminals forward the information from the source to the destination mainly using two well known methods: amplify-and-forward (AF) and decode-and-forward (DF). In AF mode, the relay terminal does not decode or demodulate, but amplifies the received observations and retransmits to the destination.

The use of relays is now being considered in cognitive radio networks for user cooperation [10], [11]. In [10] the cognitive transmitter is assisted by a group of relays only when the primary user is absent. In [11] relays are used in a cognitive radio network in which the performance of relay assisted transmission is compared with direct transmission. In this case only one primary transmitter-receiver pair is considered for comparison.

In this paper we consider a secondary system deployed in a field of primary receivers. The secondary system consists of source, relay and destination terminals where the source can communicate to the destination terminal either directly (direct mode) or using the relay (relaying mode). Our primary interest is in comparing the ability of the cognitive relay and the direct link to provide reasonable rates while co-existing with the primary users. In particular, the potential for the relay system to reduce interference by reducing transmit power is evaluated. There is certainly support for the idea that relay systems will produce less interference due to lower transmit powers [12], [13]. In [12], for example, this assumption seems to be assumed correct without any evaluation. However, such a conclusion may not always be valid and it is useful to take a detailed look at the simple case of a single cognitive radio relay surrounded by primary receivers. The comparison of the relaying and direct modes can be made in several ways, and for fairness the two modes must operate under equivalent conditions. Hence, we consider the following scenarios:

- If the SNR at the destination is the same for both modes, how do transmit power and interference vary?

- If the capacity is the same for both modes, how do transmit power and interference vary?

- If the interference is the same for both modes, how do transmit power and capacity vary?

In order to evaluate these scenarios we derive analytical expressions for the outage probability of both modes including pathloss, shadowing and multipath fading, and validate the analytical results by simulation. We also derive analytical expressions for the maximum interference caused by both modes to the primary receivers. These derivations enable a fair comparison between the two modes but do not lead to convenient closed form results. Hence, a numerical approach is taken to some of the computations. Due to the iterative nature of some of the analysis, these numerical solutions are essential and it is difficult to obtain accurate answers via simulation.

\section{SySTEM MODEL}

We consider a single secondary system coexisting in a field of primary receivers as shown in Fig. 1. The primary receivers 
are denoted as $P_{i}(i=1,2, \ldots)$ and their spatial distribution follows a Poisson point process with a density parameter, $\lambda_{p}$, which denotes the average number of primary users per unit area [4]. The secondary system consists of the source $\left(S_{c}\right)$, relay $\left(R_{c}\right)$, and destination $\left(D_{c}\right)$ terminals and each of them is equipped with a single antenna. $S_{c}$ can communicate to $D_{c}$ either directly (direct mode) or using the relay, $R_{c}$, (relaying mode). In this paper we will compare the performance of these two options. We assume that $S_{c}$ is located at the origin, $(0,0)$, and $D_{c}$ is located $2 D$ meters to the right at $(2 D, 0)$. For the secondary relay the optimal location $(D, 0)$, midway between $S_{c}$ and $D_{c}$, is chosen so that the full benefits of relaying can be assessed [14].

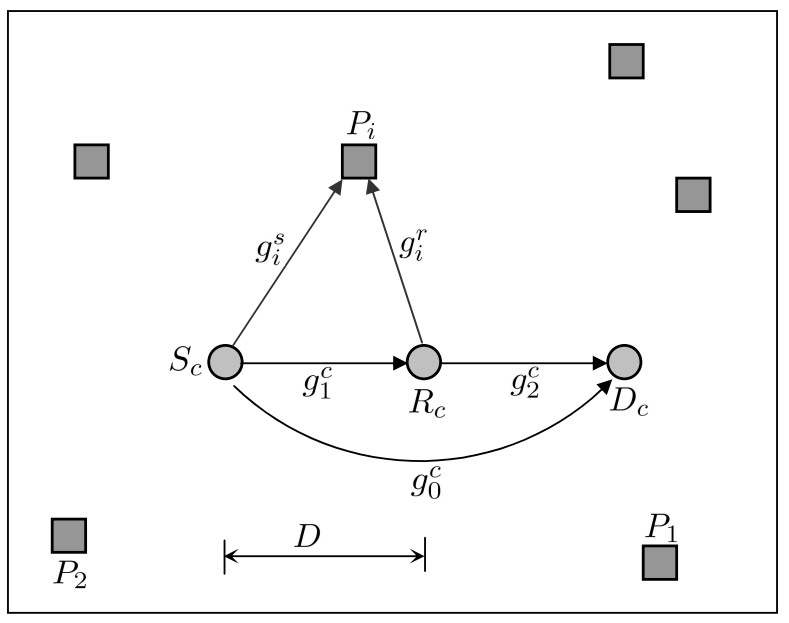

Fig. 1. Cognitive radio system model.

The complex channel gains for the source-to-destination $\left(S_{c} \rightarrow D_{c}\right)$, source-to-relay $\left(S_{c} \rightarrow R_{c}\right)$, and relay-to-destination $\left(R_{c} \rightarrow D_{c}\right)$ links are given by $g_{0}^{c}, g_{1}^{c}$, and $g_{2}^{c}$, respectively. The instantaneous channel gains from $S_{c}$ and $R_{c}$ to the $i$ th primary receiver are denoted by $g_{i}^{s}$ and $g_{i}^{r}$, respectively. In relaying mode, we assume for simplicity that there is no direct link between $S_{c}$ and $D_{c}$. During the first hop, the source terminal transmits to the relay. In the second hop, the relay terminal transmits the amplified signal from the first hop to the destination. We also assume that in relaying mode both $S_{c}$ and $R_{c}$ use a transmit power of $p_{r}$, and in the direct mode $S_{c}$ uses a power of $p_{d}$. We also assume that the secondary relay terminal assists in the communication with the destination terminal using the AF method. In this method, without decoding or demodulation the relay terminal, $R_{c}$ amplifies the received observation corresponding to the signal from the source by a factor, $a$, and retransmits it to the destination. In this paper, we use a time division multiple access (TDMA) scheme, which implies that in any time slot, $S_{c}$ or $R_{c}$ is the only interference source to the primary receivers. Note that there are two key assumptions in the relaying mode of the system model: half duplex relaying and no direct link from source to destination. If these assumptions are relaxed then the relaying mode capacity will increase and the potential for interference reduction will grow. Nevertheless, the simple structure considered here is well-established and leads to insights into the interference properties of cognitive radio relays.
We use a realistic channel model which includes pathloss, shadowing and multipath fading. Channel gains are given by

$$
g_{j}^{z}=\sqrt{A L_{j}^{z}\left(r_{j}^{z}\right)-\gamma} h_{j}^{z}
$$

where $z \in\{c, s, r\}, A$ is a pathloss-related constant, $r_{j}$ is the distance between the transmitter and the receiver of the corresponding channel, $\gamma$ is the pathloss exponent ranging from 2 to 5 [15], and $L_{j}^{z}$ and $h_{j}^{z}$ are random variables which model the effects of shadowing and multipath fading. We assume that the shadowing factors, $L_{j}^{z}$, are mutually independent, following a lognormal distribution with zero mean and a standard deviation, $\sigma_{s f}$, ranging from 5 to $12 \mathrm{~dB}$ [16]. We further assume that the fading factors, $h_{j}^{z}$, are frequencyflat and zero mean circularly symmetric complex Gaussian (ZMCSCG) random variables of unit variance.

Consider the cognitive radio system in Fig. 1. In direct mode, $D_{c}$ receives a signal from $S_{c}$ in every time slot. The signal received by $D_{c}$ in this mode can be given by

$$
y_{d}=g_{0}^{c} \sqrt{p_{d}} x+n_{d},
$$

where $n_{d}$ is the noise at $D_{c}$ and $x$ is the normalized transmit symbol. In relaying mode, $D_{c}$ receives a signal from $S_{c}$ through $R_{c}$ in every other time slot and the received signal at $D_{c}$ after the two hops is given by:

$$
y_{r}=g_{2}^{c} g_{1}^{c} a \sqrt{p_{r}} x+g_{2}^{c} a n_{r}+n_{d} .
$$

In (3), $n_{r}$ is the noise at $R_{c}$. The noise at $R_{c}$ and $D_{c}$ is modeled as ZMCSCG with $E\left\{n_{r} n_{r}^{\dagger}\right\}=\sigma_{r}^{2}$, and $E\left\{n_{d} n_{d}^{\dagger}\right\}=$ $\sigma_{d}^{2}$.

The relay amplification factor, $a$, is determined by using the power constraint, $\mathrm{E}\left\{\left|a\left(g_{1}^{c} \sqrt{p_{r}} x+n_{r}\right)\right|^{2}\right\}=p_{r}$ and assuming instantaneous knowledge of the $S_{c} \rightarrow R_{c}$ link is known at $R_{c}$. Thus, the amplification factor can be calculated as

$$
a=\sqrt{\frac{p_{r}}{\left|g_{1}^{c}\right|^{2} p_{r}+\sigma_{r}^{2}}},
$$

where the expectation is taken over $x$ and $n_{r}$. This power constraint ensures that on average the transmit power at $R_{c}$ is $p_{r}$.

\section{Outage Probability}

In this section, we calculate the outage probability of the direct and relaying mode based on the signal-to-noise ratio $(S N R)$ at the receiver.

\section{A. Direct Mode}

The instantaneous $S N R$ of the direct mode, $S N R_{d}$, can be calculated from (1) and (2) as,

$$
S N R_{d}=\frac{p_{d} A(2 D)^{-\gamma} L_{0}^{c}\left|h_{0}^{c}\right|^{2}}{\sigma_{d}^{2}} .
$$

The system is considered to be in outage if the $S N R$ at $D_{c}$ is lower than some threshold $S N R$ value, $t_{s}$. Hence, the outage probability of the direct mode can be given as,

$$
P_{\text {out }}^{d}\left(t_{s}\right)=\operatorname{Pr}\left[\frac{p_{d} A(2 D)^{-\gamma} L_{0}^{c}\left|h_{0}^{c}\right|^{2}}{\sigma_{d}^{2}}<t_{s}\right] \text {. }
$$


Since $L_{0}^{c}$ is lognormal and $h_{0}^{c}$ is a ZMCSCG random variable with unit variance, the probability density functions (PDFs) of $L_{0}^{c}$ and $\left|h_{0}^{c}\right|^{2}$ can be written as follows [17]:

$$
f_{L}(x)=\frac{\xi}{\sqrt{2 \pi} \sigma_{s f} x} e^{-\frac{\left(10 \log _{10} x\right)^{2}}{2 \sigma_{s f}^{2}}},
$$

where $\xi=\frac{10}{\ln 10}$ and $f_{|h|^{2}}(x)=e^{-x}$. Then, calculating the joint PDF of $L_{0}^{c}$ and $\left|h_{0}^{c}\right|^{2}, f_{L|h|^{2}}(x)$, and using (6), the outage probability can be computed as

$$
\begin{aligned}
P_{\text {out }}^{d}\left(t_{s}\right) & =\int_{0}^{k_{1}} f_{L|h|^{2}}(x) d x \\
& =1-\int_{0}^{\infty} \frac{\xi}{\sqrt{2 \pi} \sigma_{s f} l} e^{-\frac{\left(\operatorname{lolog}_{10}(l)\right)^{2}}{2 \sigma_{s f}^{2}}-\frac{k_{1}}{l}} d l
\end{aligned}
$$

where $k_{1}=t_{s} \sigma_{d}^{2} /\left(p_{d} A(2 D)^{-\gamma}\right)$. A closed form expression for the integral in (8) is difficult to obtain but it can easily be evaluated numerically.

\section{B. Relaying Mode}

The instantaneous $S N R$ of the relaying mode, $S N R_{r}$, can be calculated as,

$$
\begin{aligned}
S N R_{r} & =\frac{p_{r}^{2} A^{2} D^{-2 \gamma} L_{1}^{c}\left|h_{1}^{c}\right|^{2} L_{2}^{c}\left|h_{2}^{c}\right|^{2}}{p_{r} A D^{-\gamma} L_{2}^{c}\left|h_{2}^{c}\right|^{2} \sigma_{r}^{2}+p_{r} A D^{-\gamma} L_{1}^{c}\left|h_{1}^{c}\right|^{2} \sigma_{d}^{2}+\sigma_{r}^{2} \sigma_{d}^{2}} \\
& \triangleq \frac{L_{1}^{c}\left|h_{1}^{c}\right|^{2} L_{2}^{c}\left|h_{2}^{c}\right|^{2}}{c_{2} L_{2}^{c}\left|h_{2}^{c}\right|^{2} / c_{1}+c_{3} L_{1}^{c}\left|h_{1}^{c}\right|^{2} / c_{1}+c_{4} / c_{1}},
\end{aligned}
$$

where $c_{1}=p_{r}^{2} A^{2} D^{-2 \gamma}, c_{2}=p_{r} A D^{-\gamma} \sigma_{r}^{2}, c_{3}=p_{r} A D^{-\gamma} \sigma_{d}^{2}$ and $c_{4}=\sigma_{r}^{2} \sigma_{d}^{2}$. The outage probability of the relaying mode can be given as,

$$
\begin{aligned}
P_{\text {out }}^{r}\left(t_{s}\right) & =\operatorname{Pr}\left[S N R_{r}<t_{s}\right]=\mathrm{E}\left\{\operatorname{Pr}\left[S N R_{r}<t_{s} \mid L_{1}^{c}, L_{2}^{c}\right]\right\} \\
& \triangleq \mathrm{E}\left\{P_{\text {out }}^{r}\left(t_{s} \mid L_{1}^{c}, L_{2}^{c}\right)\right\} .
\end{aligned}
$$

The conditional probability, $P_{\text {out }}^{r}\left(t_{s} \mid L_{1}^{c}, L_{2}^{c}\right)$, is given by,

$$
\begin{aligned}
& P_{\text {out }}^{r}\left(t_{s} \mid L_{1}^{c}, L_{2}^{c}\right) \\
& =\mathrm{P}\left\{b_{1}\left|h_{1}^{c}\right|^{2}\left|h_{2}^{c}\right|^{2}-b_{2}\left|h_{2}^{c}\right|^{2}-b_{3}\left|h_{1}^{c}\right|^{2} \leqslant b_{4} \mid L_{1}^{c}, L_{2}^{c}\right\},
\end{aligned}
$$

where $b_{1}=L_{1}^{c} L_{2}^{c}, \quad b_{2}=t_{s} c_{2} L_{2}^{c} / c_{1}, b_{3}=t_{s} c_{3} L_{1}^{c} / c_{1}$ and $b_{4}=t_{s} c_{4} / c_{1}$. The fading terms, $\left|h_{1}^{c}\right|^{2}$ and $\left|h_{2}^{c}\right|^{2}$, are independent and both have the exponential distribution. Then, considering the region where $S N R_{r}<t_{s}$, the conditional probability given in (11) can be calculated as,

$$
\begin{aligned}
& P_{\text {out }}^{r}\left(t_{s} \mid L_{1}^{c}, L_{2}^{c}\right)=\int_{0}^{b_{3} / b_{1}} \int_{0}^{\infty} f_{|h|^{2}}(w) f_{|h|^{2}}(v) d w d v \\
& \quad+\int_{b_{3} / b_{1}}^{\infty} \int_{b_{2} / b_{1}}^{\frac{b_{4}+b_{2} v}{b_{1} v-b_{3}}} f_{|h|^{2}}(w) f_{|h|^{2}}(v) d w d v \\
& \quad+\int_{b_{3} / b_{1}}^{\infty} \int_{0}^{b_{2} / b_{1}} f_{|h|^{2}}(w) f_{|h|^{2}}(v) d w d v
\end{aligned}
$$

The integrals in (12) can be evaluated and simplified to give,

$$
\begin{aligned}
& P_{\text {out }}^{r}\left(t_{s} \mid L_{1}^{c}, L_{2}^{c}\right)= \\
& 1-2 e^{-\frac{\left(b_{3}+b_{2}\right)}{b_{1}}} \sqrt{\frac{b_{4}}{b_{1}}+\frac{b_{2} b_{3}}{b_{1}^{2}}} K_{1}\left(2 \sqrt{\frac{b_{4}}{b_{1}}+\frac{b_{2} b_{3}}{b_{1}^{2}}}\right),
\end{aligned}
$$

where $K_{1}(x)$ is a modified Bessel function of the second kind. The variables $L_{1}^{c}$ and $L_{2}^{c}$ have lognormal distributions, and hence, the outage probability in (10) can be evaluated as,

$$
\begin{array}{r}
P_{\text {out }}^{r}\left(t_{s}\right)=\int_{0}^{\infty} \int_{0}^{\infty} P_{\text {out }}^{r}\left(t_{s} \mid L_{1}^{c}, L_{2}^{c}\right) \frac{\xi^{2}}{2 \pi \sigma_{s f}^{2} l t} \\
e^{-\frac{\left(10 \log _{10}(l)\right)^{2}}{2 \sigma_{s f}^{2}}-\frac{\left(10 \log _{10}(t)\right)^{2}}{2 \sigma_{s f}^{2}}} d l d t .
\end{array}
$$

Again, a closed form expression for the integrals in (14) is difficult to obtain and has to be evaluated numerically.

\section{CAPACITY Distributions OF THE SECONDARY SYSTEM}

The instantaneous capacity of the secondary system in direct mode and relaying mode can be given by [18] as

$$
C_{d}=\log _{2}\left(1+S N R_{d}\right)
$$

and

$$
C_{r}=\frac{1}{2} \log _{2}\left(1+S N R_{r}\right)
$$

respectively. The factor of $1 / 2$ in (16) accounts for the fact that in relaying mode the information is conveyed to the destination terminal over two time slots [8].

Using the results given in Sec. III (equations (8) and (14)) the capacity distribution of the direct mode, $\mathrm{P}\left\{C_{d}<t_{c}\right\}$ and the relaying mode, $\mathrm{P}\left\{C_{r}<t_{c}\right\}$ can be obtained as

$$
\mathrm{P}\left\{C_{d}<t_{c}\right\}=P_{\text {out }}^{d}\left(2^{t_{c}}-1\right)
$$

and

$$
\mathrm{P}\left\{C_{r}<t_{c}\right\}=P_{\text {out }}^{r}\left(2^{2 t_{c}}-1\right) .
$$

To compare the direct and relaying mode, we obtain the powers, $p_{d}$ and $p_{r}$, needed for the same outage probability or for the same capacity performance. Once these power values are obtained, we calculate the maximum interference caused by secondary transmissions with these powers to the primary receivers. Therefore, the following section calculates the maximum interference power distributions for both modes.

\section{MAXimum Interference Distribution}

Consider the direct mode in which $S_{c}$ transmits signals in every time slot with power, $p_{d}$. The interference power present at the primary receiver, $P_{i}$, is given by

$$
p_{i}^{s}=A p_{d} L_{i}^{s}\left|h_{i}^{s}\right|^{2}\left(r_{i}^{s}\right)^{-\gamma}
$$

where $L_{i}^{s},\left|h_{i}^{s}\right|^{2}$ and $r_{i}^{s}$ are independent random variables. The distribution of $L_{i}^{s}$ is given in (7) and $\left|h_{i}^{s}\right|^{2}$ has exponential distribution. For the distribution of $r_{i}^{s}$, we begin by assuming that $S_{c}$ only interferes with primary receivers within a distance of $V$. Then, according to the property of Poisson point process, the $\left(r_{i}^{s}\right)^{2}$ variables have identical uniform distributions within $\left[0, V^{2}\right]$ [4]. Note that in numerical work it is sometimes necessary to employ a minimum distance between the primary receivers and the source/relay to prevent the cognitive radio interference from exploding. However, simulations indicate that such a manipulation is not necessary as the results 
are insensitive to the presence of this minimum distance. Therefore, for simplicity we assume that $r_{i}^{s} \geqslant 0$ and $r_{i}^{r} \geqslant 0$. Hence, the PDF of $\left(r_{i}^{s}\right)^{\gamma}$ can be given as,

$$
f_{r_{i}^{\gamma}}(x)=\frac{2}{\gamma V^{2}} x^{2 / \gamma-1} \text { for } 0 \leqslant x<V^{\gamma} .
$$

Now, if we denote $p_{\max }^{d}=\max \left(p_{i}^{s}\right)$ as the maximum interference produced by $S_{c}$ to the primary receivers, the distribution of $p_{\max }^{d}$ can be found using the same procedure as in [4]. Hence, we obtain

$$
F_{p_{\text {max }}^{d}}(x)=\exp \left(-\lambda_{p} \pi V^{2}\left(1-F_{p_{i}^{s}}(x)\right)\right),
$$

where $F_{p_{i}^{s}}(x)$ is the CDF of $p_{i}^{s}$. Then, using the relation

$$
V^{2}\left(1-F_{p_{i}^{s}}(x)\right)=V^{2} F_{\left(p_{i}^{s}\right)^{-1}}\left(x^{-1}\right),
$$

given in [4] and letting $V \rightarrow \infty$, we obtain

$$
\begin{aligned}
V^{2} & \left(1-F_{p_{i}^{s}}(x)\right) \\
& \rightarrow\left(\frac{A p_{d}}{x}\right)^{2 / \gamma} \Gamma\left(\frac{2}{\gamma}+1\right) \exp \left(\frac{2 \sigma_{s f}^{2}}{\xi^{2} \gamma^{2}}\right) .
\end{aligned}
$$

In (23), $\Gamma(x)$ is the gamma function. Substituting (23) into (21), the maximum interference distribution can be given as

$$
\begin{aligned}
& F_{p_{\text {max }}^{d}}(x) \\
& \quad=\exp \left(-\lambda_{p} \pi\left(\frac{A p_{d}}{x}\right)^{2 / \gamma} \Gamma\left(\frac{2}{\gamma}+1\right) \exp \left(\frac{2 \sigma_{s f}^{2}}{\xi^{2} \gamma^{2}}\right)\right) .
\end{aligned}
$$

In relaying mode, $S_{c}$ transmits during the first time slot and $R_{c}$ transmits during the next time slot, both with power, $p_{r}$. Let the random variables, $p_{\max }^{r s}$ and $p_{\max }^{r r}$ be the maximum interference caused by $S_{c}$ and $R_{c}$, respectively. We assume that $p_{\max }^{r s}$ and $p_{\max }^{r r}$ are mutually independent so that the problem separates into two independent problems of the form given in (24). The independent assumption is an approximation only. Note that $p_{\max }^{r s}$ and $p_{\max }^{r r}$ depend on fast fading, shadowing and distance variables. The fast fading is exactly independent and the shadowing is usually reasonably independent at moderate separations, especially in urban environments. The distance variables are more complex to interpret. However, with two out of the three variables having zero or small correlations, the assumption seems reasonable and simulations also support the model yielding negligible correlation coefficients. Hence, the maximum interference power distribution in relaying mode can be given as

$$
\begin{aligned}
& F_{p_{\text {max }}^{r}}(x)=F_{p_{\text {max }}^{r s}}(x) \times F_{p_{\text {max }}^{r r}}(x) \\
& \quad=\exp \left(-2 \lambda_{p} \pi\left(\frac{A p_{r}}{x}\right)^{2 / \gamma} \Gamma\left(\frac{2}{\gamma}+1\right) \exp \left(\frac{2 \sigma_{s f}^{2}}{\xi^{2} \gamma^{2}}\right)\right) .
\end{aligned}
$$

Comparing (24) and (25), we see that the interference caused by both modes will be equal when $p_{r}=2^{-\gamma / 2} p_{d}$. Thus, the relaying mode will only have less interference compared to the direct mode when $p_{r}<2^{-\gamma / 2} p_{d}$. For typical values of $\gamma$ in $(2,5)$, this implies the range of values from $p_{r}<1 / 2 p_{d}$ to $p_{r}<0.17 p_{d}$ in order for interference reduction to take place with relaying.

\section{RESUlts And Discussions}

In this section we validate the results derived in sections III - V and these are then used to compare the interference produced by the direct and relaying modes. In all the results given, we let $D=50 \mathrm{~m}, \lambda_{p}=0.01$ users $/ \mathrm{m}^{2}$ and $A=1$. Furthermore, we let $\sigma_{r}^{2}=\sigma_{d}^{2}=1$ so that $p_{d}$ and $p_{r}$ correspond to the transmit $S N R\left(S N R_{T X}\right)$ of the direct and relaying modes, respectively. Similarly, the maximum interference powers received by the primary receivers are denoted by $p_{\max }^{d}$ and $p_{\max }^{r}$. Assuming that the primary receivers have unit power noise terms, then $p_{\max }^{d}$ and $p_{\max }^{r}$ correspond to interference-to-noise ratios ( $I N R \mathrm{~s})$.

Most of the analytical results derived depend on the outage probabilities given in (8) and (14). Therefore, we first validate the results given in (8) and (14) by using Monte Carlo simulation. Figure 2 shows an excellent agreement between the analytical and simulated values of the outage probability in both direct and relaying modes.

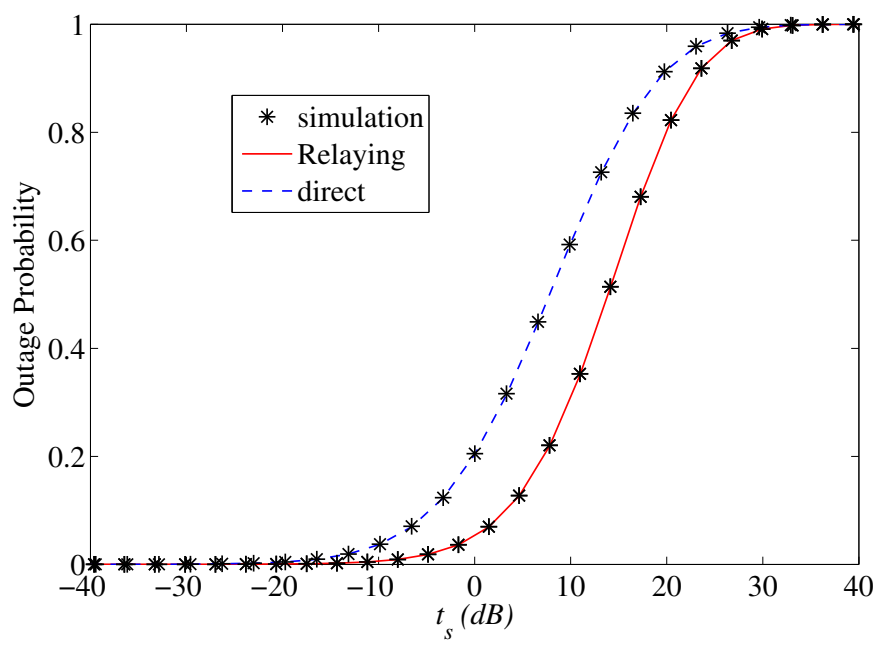

Fig. 2. Simulated and analytical values of the outage probability distribution for the direct and relaying mode with $p_{d}=p_{r}=90 \mathrm{~dB}$.

Next, we examine the maximum $I N R$ produced by the direct and relaying mode, with both modes having the same median $S N R$. For this, we first obtain the $S N R_{T X}$ values, $p_{d}$ and $p_{r}$ using (8) and (14), respectively, for a given median $S N R$ value. Then, the $S N R_{T X}$ values obtained are used in (24) and (25) to obtain the median of the maximum $I N R$. The analytical results obtained are shown in Figs. 3 and 4 for different pathloss and shadowing values, respectively. The results show that the relaying mode can have lower $I N R$ than the direct mode when there is high pathloss. The reason is that the direct mode has to boost the $S N R_{T X}$ level more than the relaying mode due to the larger distance to achieve a required $S N R$ level. Furthermore, less shadowing is favorable to the relaying mode because with less shadowing the $S N R_{T X}$ required to achieve the target $S N R$ level decreases more quickly in the relaying mode than the direct mode. Note that sometimes the $S N R_{T X}$ required by the relaying mode to achieve the same $S N R$ level is less compared to the direct mode. However, the relaying mode must have $S N R_{T X}$, $p_{r}<2^{-\gamma / 2} p_{d}$, to have lower $I N R$ than the direct mode. 
Therefore, to achieve the same median SNR level, the relaying mode can cause less $I N R$ than the direct mode only when the channel has high pathloss and low shadowing conditions.

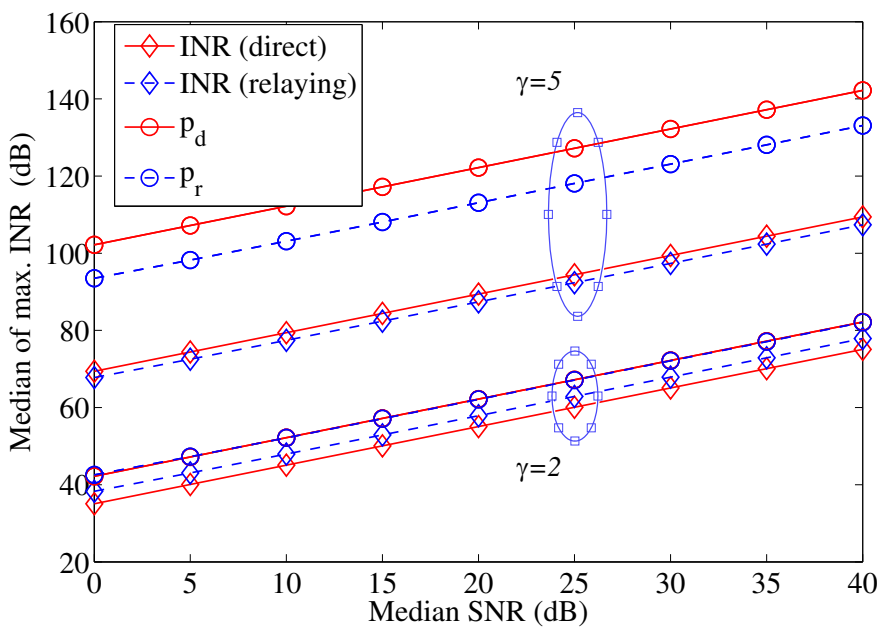

Fig. 3. The median of the maximum $I N R$ produced and the $S N R_{T X}$ required by both modes for the same median $S N R$ level with different pathloss parameters $\left(\sigma_{s f}=7.5 \mathrm{~dB}\right)$.

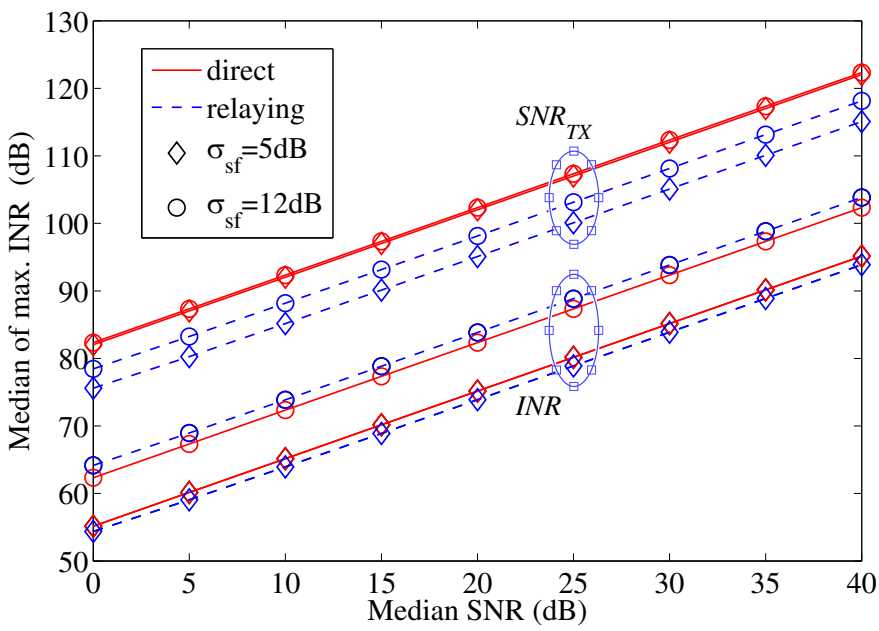

Fig. 4. The median of the maximum $I N R$ produced and the $S N R_{T X}$ required by both modes for the same median $S N R$ level with different shadowing parameters $(\gamma=4)$.

Another question of interest is, which mode generates less $I N R$ while achieving the same capacity performance. In this case we produce results with two sets of channel conditions: high pathloss $(\gamma=5)$ and low shadowing $\left(\sigma_{s f}=5 \mathrm{~dB}\right)$ and low pathloss $(\gamma=3)$ and high shadowing $\left(\sigma_{s f}=12 \mathrm{~dB}\right)$. Figure 5 shows the $I N R$ produced by both modes with the same capacity performance. Clearly, the $I N R$ produced by the relaying mode is much higher than that of the direct mode even for favorable channel conditions (high pathloss and small shadowing). The main reason is that the $1 / 2$ factor in the capacity expression forces the relaying mode to have much higher $S N R_{T X}$ than the direct mode. Further, even when the relaying mode has less $S N R_{T X}$ than the direct mode, $p_{r}$ is not less than $2^{-\gamma / 2} p_{d}$ so that the $I N R$ is greater than the direct mode.

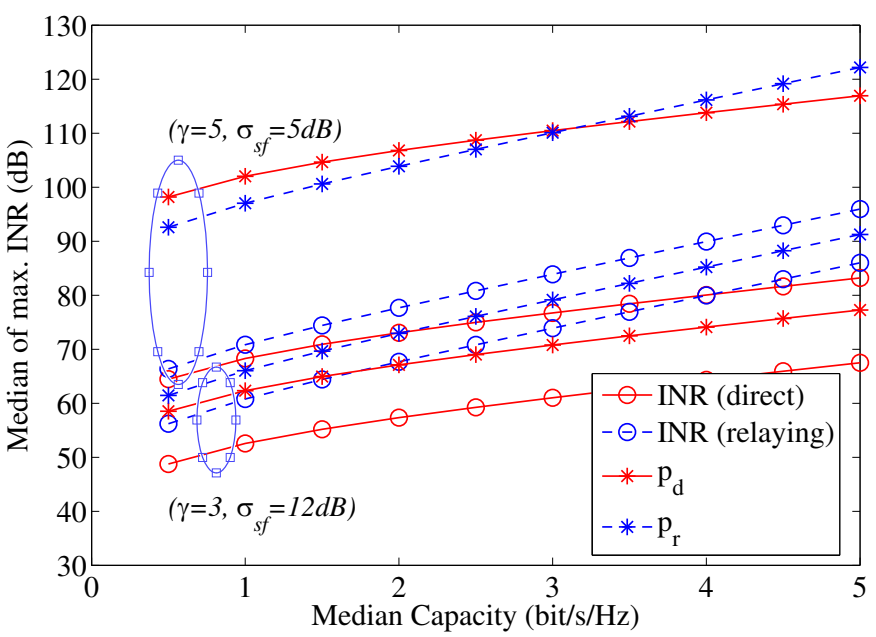

Fig. 5. The median of the maximum $I N R$ produced and the $S N R_{T X}$ required by the direct and the relaying mode for the same capacity performance with different channel conditions.

Figure 5 shows that the relaying mode usually has higher $I N R$ to the primary receivers than the direct mode if they need to achieve same capacity. From this, another question of interest is how much capacity the relaying mode has to lose compared to the direct mode to have the same $I N R$. Figure 6 shows the $I N R$ loss (ratio of the median $I N R$ of the direct mode to that of the relaying mode) against the median capacity loss (median capacity of the direct mode - median capacity of the relaying mode) of the relaying mode compared to the direct mode in a typical channel condition $\left(\gamma=4, \sigma_{s f}=7.5 \mathrm{~dB}\right)$. The results show that the capacity of the relaying mode has to be reduced by about half to have the same level of $I N R$ as the direct mode.

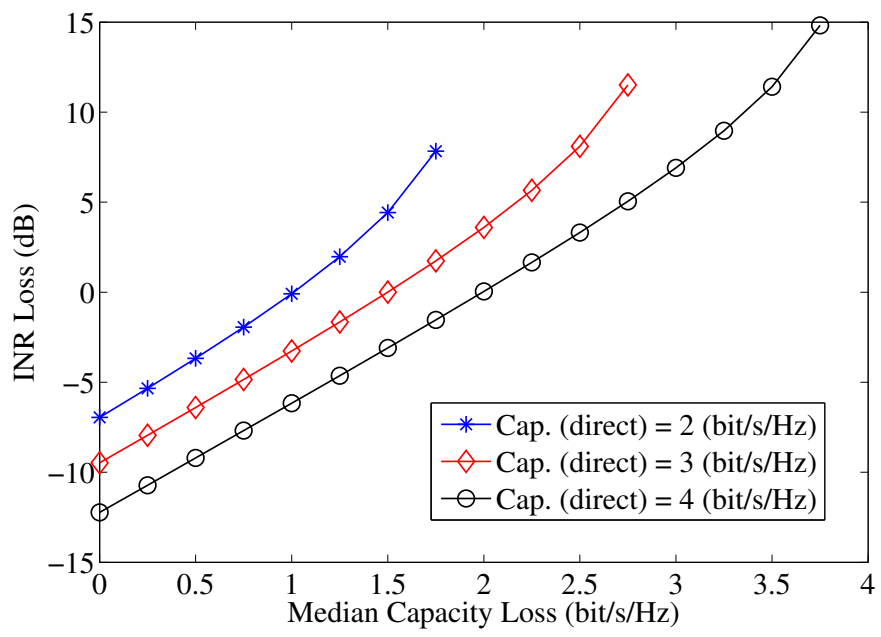

Fig. 6. INR reduction of the relaying mode vs capacity loss.

\section{CONCLUSIONS}

In this paper, we have compared the interference caused by a secondary system to the primary receivers in a cognitive radio 
network. We have considered a secondary system deployed in a Poisson field of primary receivers whose density is given by $\lambda_{p}$. We assumed that the secondary system consists of the source, relay, and destination terminal, and the source terminal can communicate with the destination terminal either directly (direct mode) or using the relay (relaying mode). We derived analytical expressions for the outage probability of both modes including pathloss, shadowing and multipath fading and validated the analytical results by simulation. We also derived analytical expressions for the maximum interference caused by both modes to the primary receivers. The results showed that to achieve the same median $S N R$ level, the relaying mode may cause less interference to the primary receivers than the direct mode in high pathloss and low shadowing conditions. However, the relaying mode has much higher interference than the direct mode if both modes have the same capacity performance. The results also showed that the capacity of the relaying mode has to be reduced by about one half to provide the same level of interference as the direct mode in a typical channel environment.

\section{REFERENCES}

[1] "Federal Communication Commission, Spectrum policy task force report," Washington DC, 2 Nov. 2002.

[2] Q. Zhao and B. Sadler, "A survey of dynamic spectrum access," IEEE Signal Processing Mag., vol. 24, no. 3, pp. 79-89, May 2007.

[3] J. Mitola and G. Maguire, "Cognitive radio: making software radios more personal," IEEE Personal Communications, vol. 6, no. 4, pp. 1318, Aug 1999

[4] X. Hong, C.-X. Wang, and J. Thompson, "Uplink cell capacity of cognitive radio networks with peak interference power constraints," in Proc. IEEE Int. Conf. on Communications, Circuits and Systems (ICCCAS), Xiamen, China, 25-27 May 2008, pp. 372-377.
[5] S. Srinivasa and S. Jafar, "Cognitive radios for dynamic spectrum access - the throughput potential of cognitive radio: A theoretical perspective," IEEE Commun. Mag., vol. 45, no. 5, pp. 73-79, May 2007.

[6] E. Wong and C. Foh, "Analysis of cognitive radio spectrum access with finite user population," IEEE Commun. Lett., vol. 13, no. 5, pp. 294-296, May 2009.

[7] C.-X. Wang, X. Hong, H.-H. Chen, and J. Thompson, "On capacity of cognitive radio networks with average interference power constraints," IEEE Trans. Wireless Commun., vol. 8, no. 4, pp. 1620-1625, April 2009.

[8] R. U. Nabar, H. Bolcskei, and F. Kneubuhler, "Fading relay channels: Performance limits and space-time signal design," IEEE J. Select. Areas Commun., vol. 22, no. 6, pp. 1099-1109, Aug. 2004.

[9] A. Sendonaris, E. Erkip, and B. Aazhang, "User cooperation diversity - part 1: System description," IEEE Trans. Commun., vol. 51, no. 11, pp. 1927-1938, Nov. 2003.

[10] K. Lee and A. Yener, "Outage performance of cognitive wireless relay networks," in Proc. IEEE Global Telecommunications Conf., San Francisco, California, USA, 27 Nov. - 1 Dec. 2006, pp. 1-5.

[11] C. Sun and K. Ben Letaief, "User cooperation in heterogeneous cognitive radio networks with interference reduction," in Proc. IEEE Int'l. Conf on Communications, Beijing, China, 19-23 May 2008, pp. 3193-3197.

[12] J. Sun, "Uplink capacity enhancement in two-hop cellular networks with limited mobile relays," in Proc. IEEE Workshop on Local and Metropolitan Area Networks, New York, NY, USA, 10-13 Jun. 2007, pp. $134-138$.

[13] K. Georgiev and D. Dimitrova, "Impact of relaying on inter-cell interference in mobile cellular networks," in Proc. European Wireless Conference, Lucca, Italy, 12-15 Apr. 2010.

[14] G. Farhadi and N. Beaulieu, "On the ergodic capacity of multi-hop wireless relaying systems," IEEE Trans. Wireless Commun., vol. 8, no. 5, pp. 2286-2291, May 2009.

[15] T. S. Rappaport and T. Rappaport, Wireless Communications: Principles and Practice (2nd Edition). Prentice Hall PTR, 2001.

[16] G. Stuber, Principles of Mobile Communications, 2nd ed. Boston, USA: Kluwer Academic Publishers, 2001.

[17] W. C. Jakes, Microwave Mobile Communications. New York, NY, USA: Wiley, 1974.

[18] M. Herdin, "MIMO amplify-and-forward relaying in correlated MIMO channels," in Proc. Int. Conf. on Inform. Commun. and Signal Processing, Bangkok, Thailand, 6-9 Dec. 2005, pp. 796-800. 